\title{
Actualización en osteonecrosis y fractura de rótula tras prótesis total de rodilla.
}

\author{
DOI: http//dx.doi.org/10.37315/SOTOCAV202028455119
}

\author{
RODRÍGUEZ COLLELL JR ${ }^{1}$, MIFSUT MIEDES $D^{1,2}$. \\ 1. DEPARTAMENTO DE SALUD CLÍNICO-MALVARROSA DE VALÈNCIA \\ 2. DEPARTAMENTO DE CIRUGÍA UNIVERSITAT DE VALÈNCIA
}

\begin{abstract}
Resumen.
Las fracturas periprotésicas de rótula son mucho más frecuentes en las prótesis de rodilla en las que la rótula ha sido reemplazada. Tienen un origen traumático en el $12 \%$ de los casos y atraumático, secundario a necrosis vascular, en el 88 $\%$ restante de los casos. Las fracturas periimplante en las que la rótula ha sido sustituida y el implante es estable con un mecanismo extensor íntegro se resuelven con un tratamiento conservador. En los casos de implante estable con fractura desplazada del polo superior o inferior de la rótula el implante se deja in situ y se repara el aparato extensor según las técnicas estándar. Si existe una disrupción del aparato extensor con implante inestable, el botón rotuliano se debe retirar y se debe reparar la continuidad del aparato extensor por medio de una patelectomía. En estos casos se desaconseja la fijación interna con cerclajes de alambre. En los casos en los que el mecanismo extensor está íntegro y el implante es inestable es suficiente con la retirada del botón rotuliano. El riesgo de infección tras la cirugía de una fractura periprotésica de rótula es elevado.
\end{abstract}

Palabras clave: osteonecrosis, patella fracture, total knee prosthesis, knee arthroplasty complications

\section{Summary.}

Periprosthetic patella fractures are much more common in knee prostheses in which the patella has been replaced. The aetiology of periprosthetic patella fractures has a traumatic origin in $12 \%$ of cases and atraumatic secondary to avascular necrosis in the remaining $88 \%$. Peri-implant fractures, in which the patella has been replaced and the implant is stable with an integral extensor mechanism, resolve with conservative treatment. In cases of stable implant, with a displaced fracture of the upper or lower pole of the patella, the implant is left in situ and the extensor apparatus is repaired according to standard techniques. If there is disruption of the extensor apparatus with an unstable implant, the patellar button should be removed and continuity of the extensor apparatus repaired with a patellectomy. In these cases, internal fixation with wire cerclages is discouraged. In cases where the extensor mechanism is intact and the implant is unstable, the removal of the patellar button is sufficient. The risk of infection after surgery for a periprosthetic patella fracture is high.

\author{
Correspondencia: \\ Correspondencia: \\ Juan Ramón Rodríguez Collell \\ rocojura@hotmail.com
}




\section{Introducción}

Los diseños iniciales de las prótesis de rodilla no sustituían la rótula y con frecuencia provocaban dolor anterior en la rodilla ${ }^{1}$. Para resolver este problema se desarrollaron los implantes rotulianos, pero con su utilización aparecieron nuevas complicaciones ${ }^{2,3}$, tales como: dolor anterior de rodilla, disrrupción del aparato extensor, desgaste y aflojamiento del componente rotuliano, subluxación rotuliana, osteonecrosis y fractura de rótula.

En 1980 con la llegada de los componentes rotulianos metálicos no cementados las complicaciones relacionadas con éstos aumentaron de forma llamativa, alcanzando el $50 \%$ del total de complicaciones de las $\mathrm{PTR}^{4}$. Por este motivo se dejaron de utilizar estos modelos, siendo actualmente los implantes rotulianos de polietileno y con múltiples tetones de fijación ${ }^{5}$.

Pero a pesar de la mejora en su diseño, las complicaciones asociadas a los componente rotulianos siguen siendo bastante frecuentes, suponiendo entre el $2 \%$ al $20 \%$ del total de las complicaciones de las PTR primarias $^{3,4,6,7,8,9,10,11}$.

La incidencia media de fractura de rótula tras PTR es del $1,19 \%$ (rango $0,15-12 \%)^{3,9}$ y constituye la segunda fractura periprotésica en frecuencia tras la fractura periprotésica femoral ${ }^{12}$. La fractura rotuliana puede aparecer tanto en rótulas no sustituidas ${ }^{8}, 1 \%$ del total, como en rótulas sustituidas, $99 \%$ del total, lo que demuestra la clara asociación entre la fractura de rótula y su sustitución protésica ${ }^{3,13}$. Según datos epidemiológicos del Registro articular de la Clínica Mayo (Mayo Clinic Joint Registry), el 0,68 \% de 12.000 artroplastias totales de rodilla primarias se vinculó con una fractura rotuliana periprotésica en un periodo de trece años ${ }^{7}$. Berry ${ }^{12}$ comunicó que la tasa de fractura rotuliana posoperatoria fue más del doble después de una artroplastia total de rodilla de revisión $(1,8 \%)$ que después de una artroplastia total de rodilla primaria $(0,7 \%)$, y que fue nueve veces más alta que la tasa de fractura intraoperatoria durante la artroplastia total de rodilla de revisión $(0,2 \%)$.

Las complicaciones asociadas a la implantación de los componentes rotulianos han provocado controversia sobre su empleo entre los cirujanos ortopédicos $^{14,15,16,17,18}$. Un primer grupo defiende la sustitución de la rótula siempre ${ }^{2,19}$, mientras que un segundo grupo son contrarios a la sustitución rotuliana ${ }^{19}$ y un tercer grupo de cirujanos son defensores de la implantación selectiva de los componentes rotulianos según los $\operatorname{casos}^{13,16,18}$. Las prótesis bicompartimentales, además de evitar las complicaciones propias de los implantes rotulianos, pueden ser ventajosas. Por una parte disminuyen la liberación de partículas de desgaste del polietileno al interior de la articulación alargando la longevidad de las PTR y por otra parte acortan el tiempo $^{16,20}$ y los costes quirúrgicos ${ }^{16}$.

\section{Material y método}

Realizamos una búsqueda bibliográfica en Pubmed (Medline, Biblioteca Nacional de Medicina de los Estados Unidos).

En primer lugar, utilizamos las siguientes palabras clave por separado y en combinación: osteonecrosis, patella fracture, total knee prosthesis, knee arthroplasty complications, en trabajos publicados en los últimos 10 años. Se tomaron en consideración aquellos artículos escritos en inglés y en español. Para la obtención de los artículos más actuales se aplicó el filtro "published in the last 5 years".

Se analizaron a su vez las referencias bibliográficas de los artículos seleccionados con la finalidad de rescatar otros estudios potencialmente incluibles para la revisión.

Por último, se revisaron diversos artículos y libros relacionados directamente con la cirugía ortopédica relacionados con la patología objeto del estudio, a los que se había llegado a partir de los artículos de interés o bien a través de consulta en archivo.

\section{Etiología}

Las fracturas periprotésicas de rótula pueden ser de origen traumático $(12 \%)^{21}$ o atraumático, secundarias a necrosis avascular $(88 \%)^{3,8,9,10}$. Los traumatismos pueden ser directos (caída con golpe directo sobre la rótula) o indirectos(contracción súbita del cuádriceps). Burnett y Bourne clasificaron los factores de riesgo asociados a las fracturas de rótula en tres grupos ${ }^{22}$ :

- Un primer grupo de factores relacionados con los pacientes que incluyen la osteoporosis, el género masculino10, las artropatías inflamatorias, la obesidad (Índice de masa corporal $>30)^{4,10,23}$, un rango de movimiento amplio ${ }^{4,10,24}$, deformidad prePTR en valgo mayor de $10^{\circ 10}$, grosor rotuliano menor de $18 \mathrm{~mm}$ e inclinación o subluxación rotuliana ${ }^{11}$.

- Un segundo grupo que depende del diseño de los implantes utilizados como implantes rotulianos no cementados, cementados con un tetón grande central ${ }^{4}$, cementados con tetones pequeños múltiples o implantes femorales posteroestabilizados ${ }^{25}$. Recientemente se han desarrollado numerosos implantes no cementados, con una superficie de anclaje poroso de tantalio. Deben observarse con prudencia, dada la tasa de fractura a 2 años del $20 \%$ observada por Chan y Giori en un estudio de 30 pacientes ${ }^{5}$.

Implantes rotulianos con mayor congruencia presentan mayor estabilidad, sin embargo, su posicionamiento es técnicamente exigente, y el riesgo de desgaste mayor. Además, en caso de revisión pueden no resultar compatibles con otros modelos protésicos a diferencia de los implantes en forma de cúpula, que son universales.

- Y un tercer grupo que depende de errores de la técnica quirúrgica, como el uso inapropiado de abrazaderas rotulianas, la excesiva resección ósea de la rótula nativa que debe conservar un grosor mínimo de 11 $\mathrm{mm}^{8,25,26,27,28,29}$, la resección asimétrica de la rótula por no identificar su unión osteocondral circunferencial ${ }^{25}$, el 
sobredimensionado rotuliano que aumenta las fuerzas de reacción articulares, la malaalineación femoral con inestabilidad rotuliana asociada ${ }^{10,30}$, la modificación excesiva de la línea $\operatorname{articular}^{26}$, y una desvascularización rotuliana excesiva.

La vascularización rotuliana se ve dañada de forma significativa con el abordaje en las PTR. Si además se reseca de forma excesiva la grasa de Hoffa, lo que supone un insulto vascular a la perfusión del polo inferior rotuliano, y se libera el retináculo lateral de la rótula para mejorar el recorrido rotuliano, se aumenta en gran medida el riesgo de fractura de rótula periimplante ${ }^{31}$. Por ello, algunos cirujanos defienden durante la exposición de la rodilla, la retracción lateral de la rótula en lugar de su habitual eversión, para minimizar de esta forma la isquemia rotuliana durante la cirugía $^{32,33,34}$

\section{Diagnóstico}

La mayoría de las fracturas rotulianas periprotésicas son atraumáticas. Tan solo en el $12 \%$ de los casos hay un acontecimiento traumático relacionado. En el $88 \%$ de los casos restantes el diagnóstico se hace como parte de un seguimiento rutinario y la mayoría ocurren durante los dos primeros años tras la cirugía de $\mathrm{PTR}^{3,35}$. Típicamente se presentan como dolor anterior de rodilla que se intensifica al subir o bajar escaleras. En el examen físico el dolor aumenta con la palpación rotuliana ${ }^{32}$. El diagnóstico se confirma con el estudio radiográfico anteroposterior, lateral y axial. En casos dudosos se puede confirmar con una tomografía computerizada.
La necrosis avascular provoca dolor persistente en la parte anterior de la rodilla antes de que se produzca la fractura, por lo que la gammagrafía y/o la TAC pueden ayudar en el diagnóstico, al mostrar un aumento de la densidad con esclerosis ósea. Si el implante es estable debe realizarse un seguimiento regular.

\section{Clasificación}

La clasificación más utilizada es la de Ortiguera y Berry ${ }^{7,11,35,37}$ que clasificaron las fracturas periprotésicas de rótula en 3 tipos ( Fig.1):

-Tipo 1: Mecanismo extensor intacto e implante estable (fracturas de trazo vertical o transversal no desplazada).

-Tipo 2: Ruptura del Mecanismo extensor con implante estable (2a) o inestable (2b) (Fig. 2) (fractura de trazo transversal).

-Tipo 3: Mecanismo extensor intacto e implante inestable con reserva ósea adecuada (3a) o insuficiente (3b).

El tipo 3 es el más frecuente suponiendo el $50 \%$ de los casos. El tipo I constituye el $25 \%$ y el tipo II el $20 \%$ de los $\operatorname{casos}^{3,11}$.
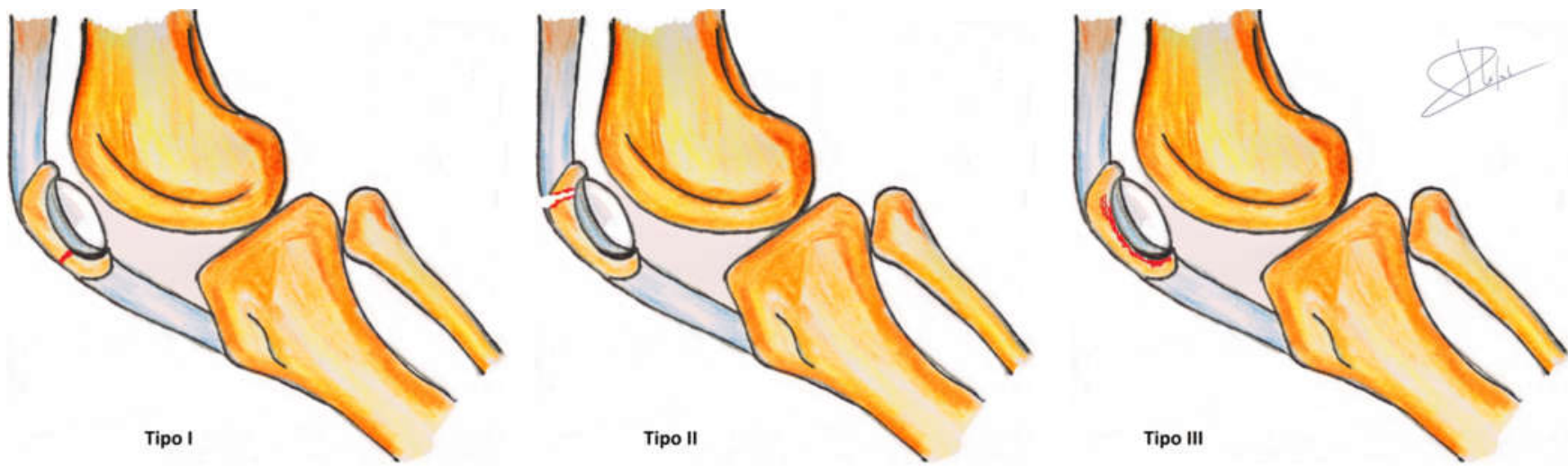

Figura 1: Clasificación de Ortiguera y Berry. 


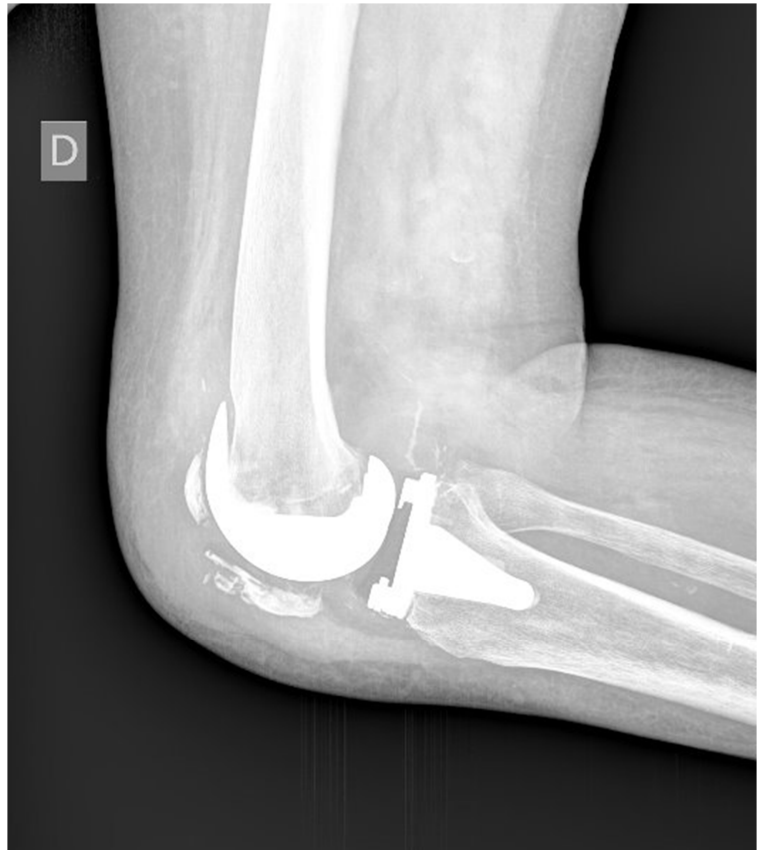

Figura 2: Fractura Tipo 2 de Ortiguera y Berry con implante inestable.

\section{Tratamiento}

El tratamiento a aplicar en las fracturas de rótula periprotésicas depende por tanto de si el aparato extensor está o no integro ${ }^{3}$, de si el botón rotuliano está o no aflojado y de la cantidad de remanente óseo ${ }^{8}$.

- En los casos en los que la rótula no ha sido sustituida se aplican las técnicas de fijación interna convencionales para las fractura de rótula. En las fracturas periimplante en las que la rótula ha sido sustituida y el implante es estable con un mecanismo extensor íntegro (Tipo I), se puede aplicar un tratamiento conservador con una férula de yeso 6 semanas $^{35}$.

- En los casos de implante estable con fractura desplazada del polo superior o inferior de la rótula (tipo Ila) el implante se deja in situ para evitar aumentar la fragilidad del hueso remanente y se repara el aparato extensor según la técnica estándar de tratamiento para las fracturas de rótula. Si existe una disrupción del aparato extensor con implante inestable (Tipo $\mathrm{Ilb}$ ), el botón rotuliano se debe retirar y se debe reparar la continuidad del aparato extensor con suturas transóseas, injertos tendinosos de isquiotibiales, patelectomía parcial o completa ${ }^{7,35,38}$ (Fig. 3) o injertos de aparato extensor de donante cuando la reparación directa no es posible, aunque con una alta tasa de complicaciones (infección y reabsorción del aloinjerto) ${ }^{39}$. Se desaconseja en estos casos la fijación interna con cerclajes de alambre ya que la tasa de no uniones alcanza el $92 \%$ de casos lo que conlleva malos resultados clínicos, puesto que los fragmentos avasculares rotulianos tienen muy poco potencial de curación ${ }^{3,9,13}$. Aunque la patelectomía completa proporciona en estos casos un buen alivio del dolor y una buena movilidad, provoca una disminución llamativa de la fuerza del cuádriceps al disminuir el momento del brazo de palanca del aparato extensor ${ }^{38}$.

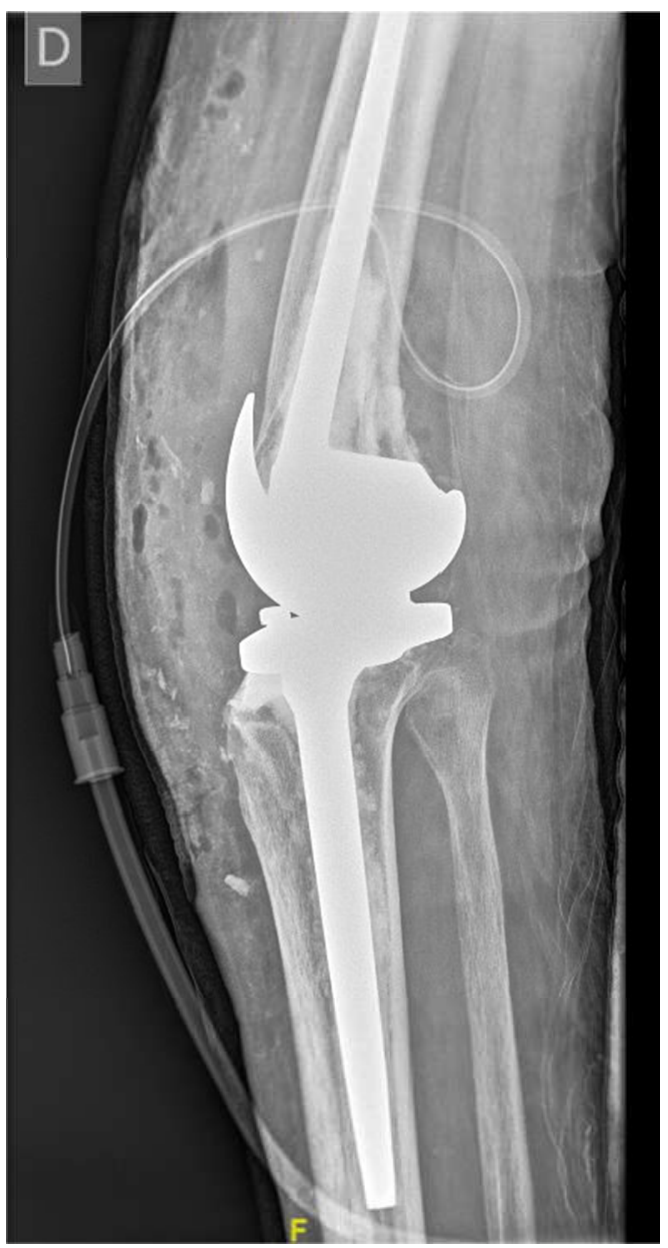

Figura 3: Patelectomía total tras fractura rotuliana asociada a osteonecrosis.

- En el tipo III Tipo 3 en que el mecanismo extensor está íntegro y el implante es inestable es suficiente el tratamiento quirúrgico con la retirada del botón rotuliano, si el remanente óseo es escaso (3a), o su sustitución si la reserva ósea es adecuada ${ }^{40}$. En cuanto al manejo del déficit de stock óseo severo, que impide la inserción de un nuevo implante rotuliano, también se dispone de diseños con implantes biconvexos o implantes con una superficie de tantalio poroso (Fig. 4). 


\section{Fracturas periprotésicas de Rótula}

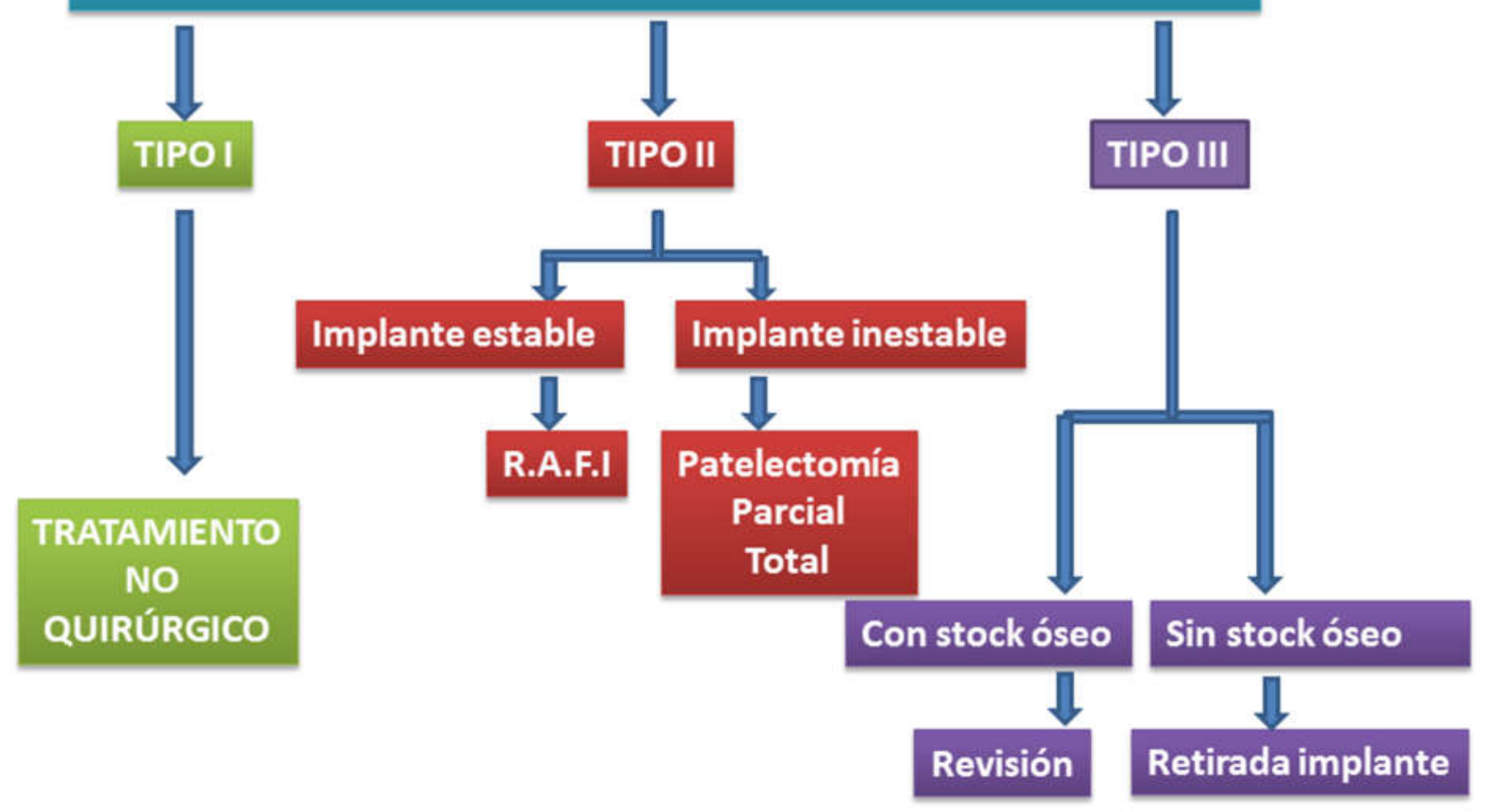

Figura 4: Algoritmo de tratamiento.

Debido a que la malposición de los componentes femoral y tibial puede estar relacionada con la fractura de rótula, el cirujano debe estar siempre preparado para llevar a cabo la revisión de los componentes protésicos femoral y tibial, aunque no hay disponibles valores de umbral validados ${ }^{41}$. En otros casos se pueden realizar procedimientos quirúrgicos similares a los utilizados para tratar la inestabilidad rotuliana en rodillas nativas.

El riesgo de infección tras la cirugía de una fractura periprotésica de rótula es alto y alcanza el 19\% de casos $^{3,9,40}$ por lo que se deben extremar las medidas para evitar esta complicación.

\section{Conclusiones}

Las fracturas periimplante en las que la rótula ha sido sustituida y el implante es estable con un mecanismo extensor íntegro se resuelven con un tratamiento conservador. En los casos de implante estable con fractura desplazada del polo superior o inferior de la rótula el implante se deja in situ y se repara el aparato extensor según las técnicas estándar. Si existe una disrupción del aparato extensor con implante inestable, el botón rotuliano se debe retirar y se debe reparar la continuidad del aparato extensor por medio de una patelectomía. En estos casos se desaconseja la fijación interna con cerclajes de alambre. En los casos en los que el mecanismo extensor está íntegro y el implante es inestable es suficiente con la retirada del botón rotuliano. 


\section{Bibliografía}

1. Insall JN, Ranawat CS, Aglietti P, Shine J.A. comparison of four models of total Knee-replacement prostheses. J Bone Joint Surg Am 1976; 58(6):754-65.

2. Lynch AF, Rorabeck CH, Bourne R B. Extensor Mechanism Complications Following Total Knee Arthroplasty. The Journal of Arthroplasty $1987 ; 2: 135-40$.

3. Chalidis BE, Tsiridis E, Tragas AA, Stavrou Z, Giannoudis PV. Management of periprosthetic patellar fractures. A systematic review of literature. Injury 2007; 38(6):714-24.

4. Healy WL, Wasilewski SA, Takei R, Oberlander M. Patellofemoral Complications Following Total Knee Arthroplasty. Correlation With Implant Design and Patient Risk Factors. The Journal of Arthroplasty $1995 ; 10: 197-201$.

5. Chan JY, Giori NJ.Uncemented metal-backed tantalum patellar components in total knee arthroplasty have a high fracture rate at midterm follow-up.JArthroplasty 2017; 2(8):2427-30.

6. Assiotis A, To K, Morgan Jones R, Pengas IP, Khan. Patellar complications following total knee arthroplasty: a review of the current literature. European Journal of Orthopaedic Surgery \& Traumatology 2019; 29(8):1605-15.

7. $\quad$ Ortiguera CJ, Berry DJ. Patellar fracture after total knee arthroplasty. J Bone Joint Surg Am 2002; 84-A(4):532-40.

8. Bourne RB. Fractures of the patella after total knee replacement. Orthopedic Clinics of North America 1999; 30: 287-91.

9. Keating EM, Haas G, OPA-C, Meding JB. Patella Fracture After Total Knee Replacements. Clin Orthop Relat Res 2003; 416:93-7.

10. Meding JB, Fish MD, Berend ME, Ritter MA, Keating E M. Predicting Patellar Failure After Total Knee Arthroplasty. Clin Orthop Relat Res 2008; 466:2769-74.

11. Putman S, Boureau F, Girard J, Migaud H, Pasquier G. Patellar complications after total knee arthroplasty. Orthopaedics \& Traumatology: Surgery \& Research 2019; 105:S43-S51.

12. Berry DJ. Epidemiology: hip and knee. Orthop Clin North Am 1999; 30:183-90.

13. Tharani R, Nakasone C, Vince KG. Periprosthetic fractures after total knee arthroplasty. J Arthroplasty 2005; 20:27-32.

14. Barrack RL, Bertot AJ, Wolfe MW, Waldman DA, Milicic M, Myers L. Patellar resurfacing in total knee arthroplasty. A prospective, randomized, double-blind study with five to seven years of follow-up. J Bone Joint Surg Am 2001; 83(9):1376-81.

15. Schiavone A, Cerciello, Del Regno C, Felici A,Vasso M. Patellar resurfacing complications in total knee arthroplasty. International Orthopaedics (SICOT) 2014; 38:313-7.

16. Lygre S, Espehaug B, Havelin L, Vollset S, Furnes O. Failure of total Knee arthroplasty with or without patella resurfacing. A study from the Norwegian Arthroplasty Register with 0-15 years of follow-up. Acta Orthopaedica 2011; 82(2):282-92.

17. Schindler O S. The controversy of patellar resurfacing in total knee arthroplasty: Ibisne in medio tutissimus?. Knee Surg Sports Traumatol Arthrosc 2012; 20:1227-44.

18. Roberts DW, Hayes TD, Tate CT, Lesko JP. Selective patellar resurfacing in total knee arthroplasty: a prospective, randomized, double-blind study. J Arthroplasty 2015; 30(2):216-22.

19. Todd C J, Penny J. Tatman MPH, Susan Mehle BS, Terence J. Gioe MD. Revision Surgery for Patellofemoral Problems Should We Always Resurface? Clin Orthop Relat Res 2012; 470:211-9.

20. Furnes O, Espehaug B, Lie S A,Vollset S E, Engesæter L B, Havelin L I et al. Early failures among 7,174 primary total knee replacements A follow-up study from the Norwegian Arthroplasty Register 1994-2000. Acta Orthop Scand 2002; 73 (2): 117-29.

21. Seijas R, Orduna J M, Castro M C,Granados N, Baliarda J, Alcantara E. Fracture of the unresurfaced patella after total knee arthroplasty: a report of two cases. Journal of Orthopaedic Surgery 2009; 17(2):251-4.

22. Bourne RB, Burnett RSJ. The consequences of not resurfacing the patella. Clin Orthop Related Res 2004; 428:166-9.

23. Rosenberg AG, Andriacchi TP, Barden R, Galante JO. Patellar component failure in cementless total knee arthroplasty. Clin Orthop 1988; 236:106-14.

24. Doo Yoo J, Kim NK. Periprosthetic fractures following total knee arthroplasty. Knee Surg Relat Res 2015; 27(1):1-9.

25. Dalury DF, Dennis DA. Extensor mechanism problems following total knee replacement. J Knee Surg 2003; 16(2):118-22.

26. JujoY, Yasui T, Nagase Y, MD, Kadono Y, Oka H, MD, Tanaka. Patellar Fracture After Total Knee Arthroplasty for Rheumatoid Arthritis. The Journal of Arthroplasty 2013; 28:40-2. 
27. Lie DTT, Gloria N, Amis A A, Lee B P H, S. J. Yeo S J, Chou S M. Patellar resection during total knee arthroplasty: effect on bone strain and fracture risk. Knee Surg Sports Traumatol Arthrosc 2005;13 : 203-8.

28. Reuben J D, Mc Donald M C, Woodard P L, Heninington L J. Effect of patella thickness on patella strain following total knee arthroplasty. J Arthroplasty 1999; 6(3):251-8.

29. Hamilton W G, Ammeen D J, Parks N L, Goyal N, Engh G A, Engh A C. Patellar Cut and Composite Thickness: The Influence on Postoperative Motion and Complications in Total Knee Arthroplasty. J Arthroplasty 2017; 32(6):1803-7.

30. Figgie HE, Goldberg VM, Figgie MP. The effect of alignment of the implant on fractures of the patella after condylar total knee arthroplasty. J Bone Joint Surg 1989; 7IA:1031-9.

31. Sayeed SA, Naziri Q, Patel YD, Boylan MR, Issa K, Mont MA. Patellar fractures following total knee arthroplasty: a review. J Long-Term Effects Med Implants 2013; 23(4):331-6.

32. Stoffel KK, Flivik G, Yates PJ, Nicholls RL. Intraosseous blood flow of the everted or laterally-retracted patella during total knee arthroplasty. Knee 2007; 14:434-8.

33. Hempfing A, Schoeniger R, Koch PP, Bischel O, Thomsen M. Patellar blood flow during knee arthroplasty surgical exposure: intraoperative monitoring by laser Doppler flowmetry. J Orthop Res 2007; 25(10):1389-94.

34. Hasegawa M, Kawamura G, Wakabayashi H, Sudo A, Uchida A. Changes to patellar blood flow after minimally invasive total knee arthroplasty. Knee Surg Sports Traumatol Arthrosc 2009; 17(10):1195-8.

35. Parvizi J, Kim K-I, Oliashirazi A, Ong A, Sharkey PF. Periprosthetic Patellar Fractures. Clin Orthop Relat Res 2006; 446:161-6.

36. Sheth NP, Pedowitz DI, Lonner JH. Periprosthetic patellar fractures. J Bone Joint Surg Am 2007; 89(10):2285-96.

37. Papalia R, Vasta S, D'Adamio S, Albo E, Maffulli N, Denaro V. Complications involving the extensor mechanism after total knee arthroplasty. Knee Surg Sports Traumatol Arthrosc. 2015; 23(12):3501-15.

38. Chang MA, Rand JA, Trousdale RT. Patellectomy after total knee arthroplasty. Clin Orthop Relat Res 2005; 440:175-7.

39. Maffulli N, Spiezia F, La Verde L, Rosa MA, Franceschi F. The Management of Extensor Mechanism Disruption After Total Knee Arthroplasty: A Systematic Review. Sports Med Arthrosc Rev 2017; 25:41-50.

40. Berend ME, Harty LD, Ritter MA, David M. Stonehouse II. Excisional Arthroplasty for Patellar Loosening in Total Knee Arthroplasty. The Journal of Arthroplasty 2003; 18:668-71.

41. Bates MD, Bryan D. Extensor Mechanism Disruption After Total Knee Arthroplasty, Am Acad Orthop Surg 2015; 23:95-106. 